\title{
Gait Unsteadiness Analysis from Motion Primitives
}

\author{
A. Sant'Anna, W. Ourique de Morais, and N. Wickström
}

\begin{abstract}
The development of intelligent ambulatory monitoring systems and smart living environments is important when considering the aging of society and its implications. This work concerns the use of human motion analysis as a tool for supporting elderly life. Movement recognition has so far been achieved through some form of template matching after manual segmentation or modeling of important features. However, previous works have failed to generalize movement and have only been able to recognize few predetermined activities. To cope with those limitations, this work suggests a new "motion language" approach. To demonstrate the viability and usefulness of this methodology, the concept of "motion primitives" was used to quantitatively analyze gait unsteadiness, which relates to physical condition and cognitive performance. The variability of stride time and temporal walk symmetry between the two feet were measured. Accelerometers were chosen as motion sensors since they offer desirable features in monitoring human movements such as response to both movement frequency and intensity, miniaturization and low power consumption. This study shows that a motion language methodology is capable of quantitatively measuring temporal gait characteristics and providing tools for continuous, unobtrusive, home-based gait analysis.
\end{abstract}

\section{INTRODUCTION}

$\mathrm{T}$ HE rapid aging of Europe's population poses new problems to be addressed in the near future. Today, about $16 \%$ of the population in Europe is aged 65 or over according to the United Nations Population Division [1]. Projections show (Fig.1) that this number will have surpassed $27 \%$ by 2050 . The increased number of elders will exert great pressure on the health care system to treat age-related problems. The percentage of economically productive individuals will subsequently decrease. To cope with limitations in available resources, the traditional health care system must shift its attention from medical facilities to patient-centered, pro-active medical assistance.

Besides softening the demands on the health-care system, enabling comfortable aging at home brings benefits to the patient and his family. The safety of the elder at home must be guaranteed by intelligent activity monitoring and sharing relevant information with informal

Manuscript received April 25th, 2008. This work is supported by the Sparbanksstiftelsen Kronan Foundation and Halmstad University within the scope of Halmstad Research School in Entrepreneurship - Health. Halmstad University, Sweden, PO Box 823, SE-301 18 HALMSTAD.

A. Sant'Anna is a PhD student at the Intelligent Systems Lab, School of Information Science, Computer and Electrical Engineering (phone: +46 35 167849; fax: +46 35 120348; e-mail: Anita.Santanna@hh.se).

W. Ourique de Morais is a PhD student at the Center for Research on Embedded Systems, School of Information Science, Computer and Electrical Engineering (e-mail: Wagner.deMorais@hh.se).

N. Wickström is with the Intelligent Systems Lab, School of Information Science, Computer and Electrical Engineering (e-mail: Nicholas.Wickstrom@hh.se).

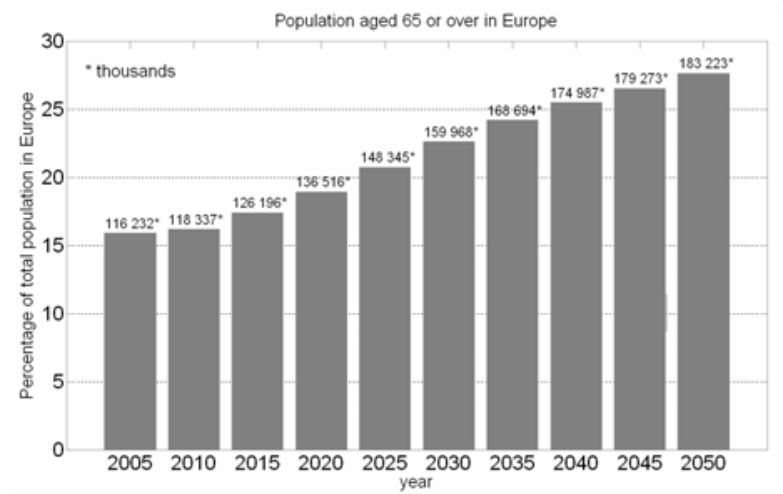

Fig.1. Percentage of population aged 65 or over in Europe.

care-takers, such as family members and friends, as well as with medical personnel. The detection and classification of movements is a powerful and intuitive way of determining the subject's health and functional status [2]. This work focuses on one particular activity, gait, which is an indicator of physical and cognitive decline in performance [3].

Several studies have focused on movement recognition for classifying gait [4] and evaluating balance [5]. These studies, however, have been based on template matching techniques and have been limited to detecting only a few predetermined activities or events. Our work presents a movement recognition technique based on decomposing an activity into elementary building blocks called "motion primitives". This methodology contributes to current research efforts by being able to generalize movements and provide higher level interpretations. The motion primitives may be organized into a "motion language" capable of describing innumerable movements from a limited number of primitives.

In order to demonstrate the viability and usefulness of the motion language methodology, this work analyzes gait unsteadiness from motion primitives. The variability of stride time and the temporal walk symmetry between the two feet were chosen as relevant parameters (Section II).

This document is organized as follows. The importance of gait analysis and related work is discussed in Section II. Favorable aspects of using accelerometers to analyze human movement, as well as relevant previous works in the area, are presented in Section III. The concept of motion language will be detailed in Section IV. Section V presents arguments on creating such motion language from accelerometer signals. Section VI presents a first study of gait unsteadiness from motion primitives. Section VII discusses different possible applications for this methodology. Finally, Section VIII concludes this paper. 


\section{GAIT ANALYSIS}

Gait is an important indicator of physical and cognitive condition. There is evidence that changes in gait are related to physical and cognitive decline due to aging [7] or illness [8]. In addition, it may help identify and quantify the risk of and elderly falling [6] and is an essential tool in the treatment of, for example, cerebral palsy [9].

Gait analysis is most commonly done by visual observation, which is subjective and depends on the clinic skills of the observer. A low-tech tool used to improve this assessment is videotaping the exam so that the clinician may take a more detailed look at the patient's walking pattern. In this scenario, the important gait parameters, such as stride time and walking speed, are measured with a stop watch, tape measure and the occasional talcum powder [10]. Nowadays, however, motion capture (mocap) systems combined with ground-force plates provide accurate description of gait patterns [11]. The drawback with this technology is that the required equipment is expensive and must be fit into an appropriate room, limiting its application.

Ideally, gait measurement systems should be easy to use, unobtrusive and mobile, to allow long term monitoring of the patient in uncontrolled environments. For that, sensors such as accelerometers and gyros are useful [12]. We chose to work with only accelerometers because of their low power consumption, which helps extend the battery life of our sensor nodes. The following section elaborates on human motion analysis systems based on accelerometers.

\section{ACCELEROMETERS}

Current enhancements in micro-electro-mechanical systems (MEMS) technology have made possible the manufacture of miniaturized, low cost accelerometers [12], good for harvesting human motion information for long periods of time and in uncontrolled environments.

Although accelerometers do not restrict the subject's movements, a downside of their mobility is that considerable thought must be put into the development of the sensor nodes. Communication, power supply and processing power are some of the issues to be considered.

When compared to video images, the accelerometer data provides localized information. In order to construct a model such as done from mocap systems, several accelerometers and gyroscopes over articulated parts of the body are needed. However, a large number of sensors is inconvenient for most long term monitoring applications. The analysis of accelerometer signals is, therefore, less intuitive. Nonetheless, accelerometers have been employed for many different purposes. Studies have been performed in which accelerometers were used to assess, among others, metabolic energy expenditure [14], physical activity [15], standing balance [5], fall detection, postural orientation and activity classification [2].

One work has studied the classification of gait patterns using data obtained from a tri-axial accelerometer. This study proposed a Gaussian Mixture Model (GMM) based classifier. One GMM per walking pattern was used to describe the following 5 patterns: flat, down slope, up slope, down stairs and up stairs [4]. Gait analysis often takes advantage of the fact that walking is a repetitive, predictable activity. The drawback of this work was limited scalability; every new pattern of interest would require a new GMM.

The following section elaborates on the concept of "motion language" and its advantages when compared to traditional movement classification techniques.

\section{Motion LANGUAGE}

A fundamental problem in detecting and recognizing human action is one of representation. Movement detection and classification has been, traditionally, achieved through some form of template matching or pattern recognition after manual segmentation or modeling of the features considered important. These techniques depend on a large collection of training examples, and labeling is labor-intensive and error-prone [16]. To cope with these draw-backs, human activity can be decomposed into building blocks which belong to an "alphabet" of elementary actions [17]. This alphabet enables the creation of a "motion language" where analogies are made between movements and words. The relationship between action and language is supported by the Mirror Neuron Theory [18] which states that the same brain mechanisms are activated when actions are performed or observed [19].

A motion language is able to generalize movement by describing innumerable concepts from different combinations of a limited number of primitives. The organization of elementary actions for classifying human movement by describing a hierarchical model has already been studied [20]. The concept of motion primitives was also explored focusing on automatically deriving vocabularies of motion modules from human motion data, taking advantage of the underlying spatial-temporal structure in motion [16]. Video image sequences have been converted into strings containing a sequence of symbols, each representing a manually determined primitive to classify five one-arm movements [21]. Also from video images (mocap database), the inference of sequential and parallel grammar rules to describe human movements has been studied [22].

\section{Motion Language From AcCelerometer Signals}

Human motion studies from accelerometers, when compared to studies using visual data, present the following advantages:

- Accelerometers can be configured into mobile, wireless, wearable systems, capable of recording information independent of location;

- Accelerometers consume very little power, therefore providing long term power autonomy for such mobile systems;

- People feel camera-like devices invade their privacy;

- The analysis of accelerometer signals may profit from analysis and processing techniques developed by other research areas such as speech recognition.

For the above mentioned reasons, this work focuses on 
the creation of a motion language from accelerometer signals. In order to successfully create this language, four tasks must be taken into consideration:

\section{A. Segmentation}

One of the greatest challenges in the process is finding the best way to segment the signal in order to convey relevant information. Ideally, the signal should be segmented according to its innate characteristics. An algorithm should be developed in order to detect repeating patterns that are, in turn, not composed of smaller patterns. A previous work made use of angular displacement and first-derivative signs to segment signals into primitives [22], but this is only one way of characterizing the signal, and it may not be the best one. Another work considered the variance characteristics of the signal [23] but the segmentation achieved was coarse and only worked when the movements involved maneuvering objects.

\section{B. Feature Extraction}

After segmentation, various features extracted from each segment of the signal are used to classify them into different symbols. The most common approach when detecting daily activities from accelerometer is to extract information from equally sized sliding windows. This does not take primitives into consideration but rather statistical information about the whole movement. For the purpose of creating a motion language, these features may be chosen with respect to their variability across the whole of the segments. The larger the variability, the better the symbols will be represented by this feature.

\section{Symbol Assignment}

The features extracted from each segment are used to differ one segment from another. Segments with similar characteristics may be assigned one symbol. These symbols may be compared to letters in the alphabet. They will be used to construct "words" and "sentences".

\section{Grammar Rules}

The grammar rules should express how symbols may be put together to form words and sentences that convey motion concepts. They may be inferred from a large collection of data. Although segmentation, feature extraction and symbol assignment may not relate directly to the physical movements, the physical limitations of our body should be reflected on these rules on syntactic and semantic levels. Syntactic analysis is concerned with deriving rules about which movements are possible, such as "our arms only bend one way." Semantic analysis considers rules about how different movements are associated. We can not, for example, "chew gum and whistle at the same time." The elaboration of these grammar rules from mocap data has been explored by Guerra-Filho et al. [22].

\section{STUDY: GAIT UNSTEADINESS ANALYSIS}

The work presented here is a study of the requirements and capabilities of the motion language methodology. It aims at demonstrating the viability and usefulness of the method by finding simple solutions to the four tasks explained in Section V (segmentation, feature extraction, symbol assignment and grammar inference) for motion analysis.

\section{A. Method}

The goal of this study was to quantitatively analyze gait unsteadiness by finding measures of variability in stride time and temporal walk symmetry between the two feet. The data was acquired with two sensor nodes with serialcommunication capabilities, each consisting of a tri-axial accelerometer, a PIC processor and an external memory (Fig.2). The sensor nodes were placed inside the sole of a pair of running shoes at the heel.

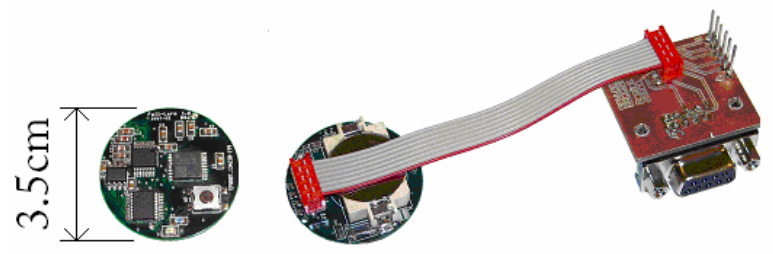

Fig. 2. One sensor node used to acquire gait data. It is composed of a triaxial accelerometer, a PIC processor and an external memory, with serialcommunication capabilities.

The acquired data corresponds to one person's three different walking patterns: normal speed walking, slow walking and limping (simulated by immobilizing the subject's right knee); sampled at $25 \mathrm{~Hz}$. Each data set corresponds to approximately 3 minutes of walking. The very beginning and end of each data set were excluded in order to consider only the more stable gait cycles. The approximate number of steps analyzed for each walking pattern is presented in Table I.

Table I. Approximate number of steps used for gait analysis.

\begin{tabular}{|c|c|c|c|}
\cline { 2 - 4 } \multicolumn{1}{c|}{} & Normal walk & Slow walk & Limp walk \\
\hline No. of steps & 175 & 142 & 85 \\
\hline
\end{tabular}

In this study, only the accelerations along the $y\left(\boldsymbol{A c c}_{\boldsymbol{y}}\right)$ and $\mathrm{Z}\left(\boldsymbol{A} \boldsymbol{c} \boldsymbol{c}_{\mathrm{z}}\right)$ axes were considered in the analysis for simplification purposes, since the walking circuit was circular. Therefore, throughout the paper, resultant acceleration refers to $\operatorname{sqrt}\left(\boldsymbol{A} \boldsymbol{c c} \boldsymbol{y}^{2}+\boldsymbol{A} \boldsymbol{c c} c_{z}^{2}\right)$, unless specified otherwise.

The segmentation of the resultant acceleration was performed with a bottom-up linear segmentation approach, as described by Keogh et al. [24]. The features extracted from each linear segment were: a measure of variability along the $y$ axis, $\boldsymbol{r}$; the tangent of the angle between the segment and the horizontal plane, $\boldsymbol{\alpha}$; average acceleration along the $y$ axis, $\boldsymbol{A} \boldsymbol{c} \boldsymbol{c}_{\boldsymbol{y}}$; and average acceleration along the $\mathrm{z}$ axis, $\boldsymbol{A} \boldsymbol{c c}_{\boldsymbol{z}}$. These features were used to k-means cluster the segments into three groups (symbols), represented by numbers 1 through 3 . Three symbols were used because they were the smallest number of symbols that could be used to satisfactorily analyze the data. Figure 3 exemplifies the correspondence between the symbols and the resultant acceleration for the normal speed walking data. The centers of the clusters found for the normal speed walking data are presented in Table II. 


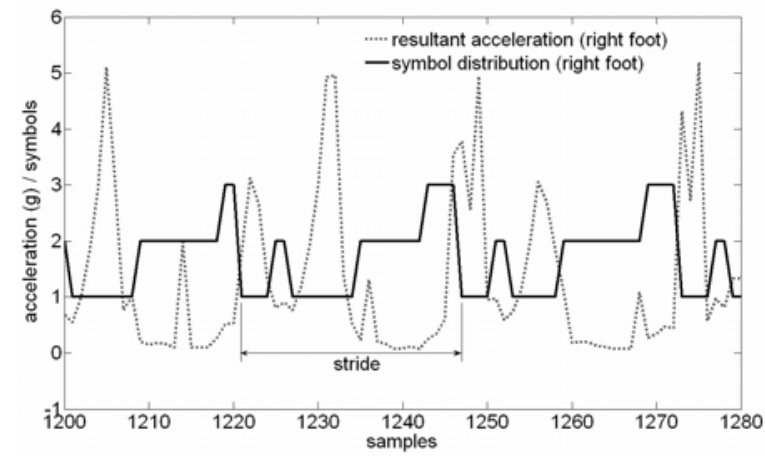

Fig. 3. Example of the correspondence between the symbols and the resultant acceleration for normal speed walking. Sampling rate: $25 \mathrm{~Hz}$.

Table II. Centers of clusters for normal speed walking data.

\begin{tabular}{|c|c|c|c|c|}
\hline \multirow{2}{*}{ Feature } & & & & \\
\hline $\mathbf{1}$ & $\boldsymbol{\alpha}$ & $\boldsymbol{r}$ & $\boldsymbol{A c c}_{\boldsymbol{y}}$ & $\boldsymbol{A c c}_{\boldsymbol{z}}$ \\
\hline $\mathbf{2}$ & -0.1253 & 0 & 0.0682 & 0.2507 \\
\hline $\mathbf{3}$ & 0.0176 & 1 & -0.0124 & -0.0010 \\
\hline
\end{tabular}

Where $\boldsymbol{r}$ is a measure of variability along the $y$ axis (1=below 0.5); $\boldsymbol{\alpha}$ is the tangent of the angle between the segment and the horizontal plane; $\boldsymbol{A} \boldsymbol{c} \boldsymbol{c}_{\boldsymbol{y}}$ is the average acceleration along the $y$ axis; and $\boldsymbol{A} \boldsymbol{c} \boldsymbol{c}_{z}$ is the average acceleration along the $z$ axis for each segment.

After assigning symbols to the segments, the raw data can be represented by strings of three symbols. The next task was to understand how these symbols relate to one another and how they can be used to derive temporal gait measurements. One simple way of representing the dynamic relationship of symbols is to consider a transition matrix, where element $(i, j)$ is the probability with which a symbol $i$ is followed by a symbol $j$. The transition matrix for the normal speed walking data is presented in Table III. These transitions can be, in turn, represented by other symbols. The codebook in Table IV assigns letters to transitions in order of decreasing occurrence.

Table III. Transition matrix for normal speed walking data.

\begin{tabular}{|c|c|c|c|}
\hline From & $\mathbf{1}$ & $\mathbf{2}$ & $\mathbf{3}$ \\
\hline $\mathbf{1}$ & 0.70 & 0.24 & 0.06 \\
\hline $\mathbf{2}$ & 0.10 & 0.68 & 0.22 \\
\hline $\mathbf{3}$ & 0.79 & 0.04 & 0.17 \\
\hline
\end{tabular}

Where element $(i, j)$ is the probability with which a symbol $i$ is followed by a symbol $j$.

Table IV. Codebook for normal speed walking data.

\begin{tabular}{|c|c|c|c|}
\hline From & 1 & 2 & 3 \\
\hline 1 & $A$ & $C$ & $G$ \\
\hline 2 & $F$ & $B$ & $E$ \\
\hline 3 & $D$ & $I$ & $H$ \\
\hline
\end{tabular}

Where letters are assigned to transitions in order of decreasing occurrence.

By visually analyzing Fig.4, one can identify a repeating stride cycle that starts with a transition $\boldsymbol{D}$. The stride time was, therefore, determined by calculating the time elapsed between two such transitions and computing the most frequent values around the expected stride time (1s).

The temporal symmetry between the two feet, SI, was estimated by how similar the stride times were (1) [25],

$$
S I=\frac{T_{R}-T_{L}}{\frac{1}{2}\left(T_{R}+T_{L}\right)} \cdot 100
$$

where $\boldsymbol{T}_{\boldsymbol{R}}$ is the average stride time for the right foot and $\boldsymbol{T}_{\boldsymbol{L}}$ is the average stride time for the left foot. The variability in stride time was estimated by the standard deviation of stride time for each foot.

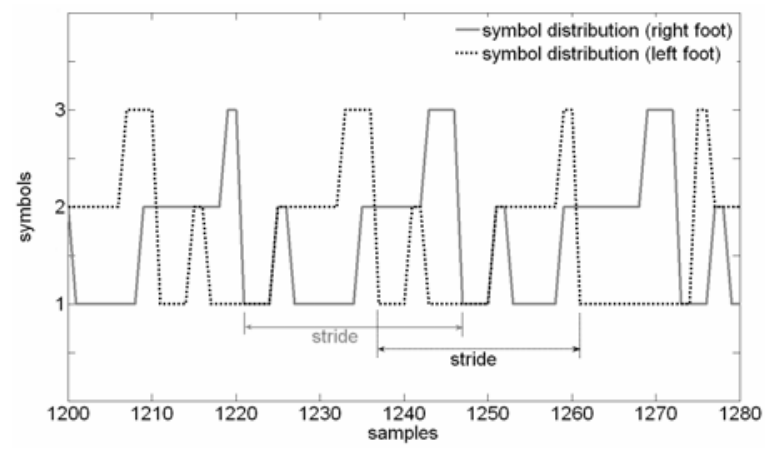

Fig. 4. Symbolic representation of normal speed walking data. Notice that there is a transition from symbol 3 to 1 (transition $D$ ) when a stride cycle begins. Sampling rate: $25 \mathrm{~Hz}$.

\section{B. Results}

The values found for the normal and slow walking data are presented as histograms in Fig.5 and Fig.6, respectively. According to the chosen measures, there is great symmetry between the two feet for both normal speed and slow walking. The variability of stride time is greater for slow walking than for normal speed walking. This is explained by the fact that the subject experienced a decrease in balance when walking slowly and had to make a conscious effort to keep a steady walking speed.
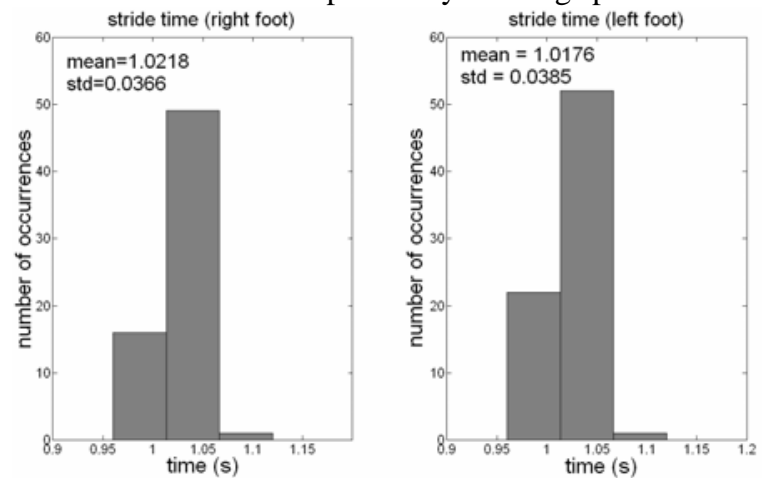

Fig. 5. Histogram of stride time, in seconds, for normal speed walking data. Approximately $40 \%$ of total data.
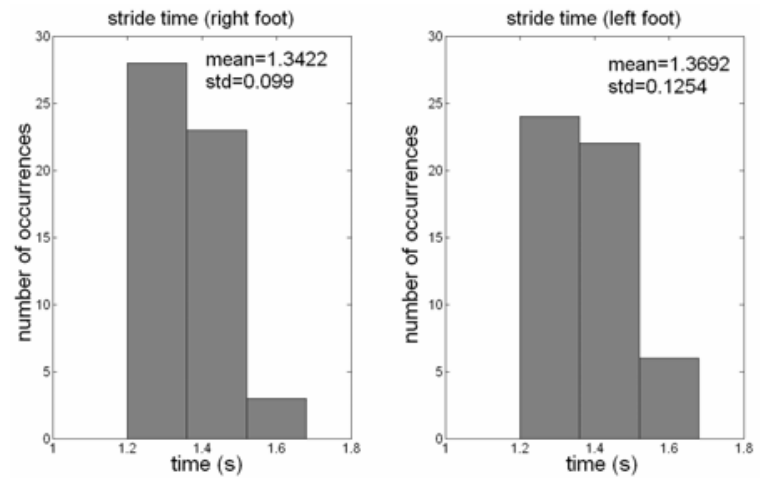

Fig. 6. Histogram of stride time, in seconds, for slow walking data. Approximately $38 \%$ of total data.

Although a repetitive cycle could be detected in the limp walking symbolic data (Fig.8), the same method was insufficient to determine the limp walking speed because 
there were no expressive peaks in the stride time histogram. The asymmetry of the limp walk is well seen in Fig.7 and Fig.8.
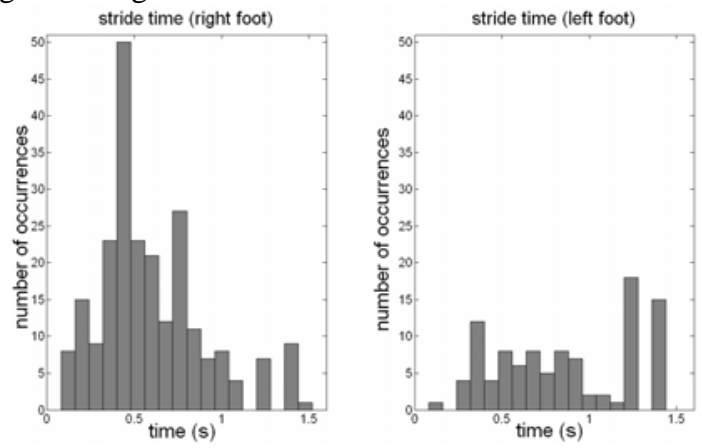

Fig. 7. Histogram of stride time, in seconds, for limp walking data.
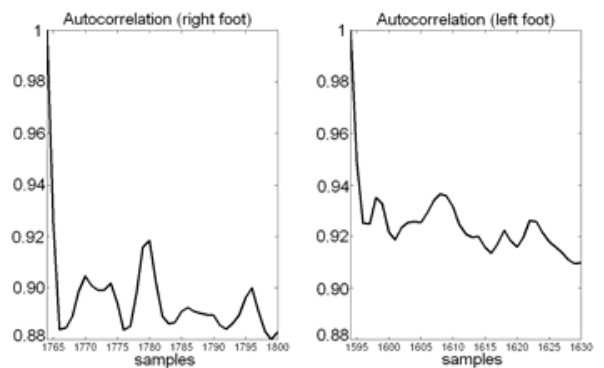

Fig.8. Autocorrelation of symbolic data for the limp walking data. Notice there are repetitive cycles of symbols for both feet but the cycles could not be detected in the same manner as for the normal walking and slow walking data.

Besides peaks of recurrent stride times around the expected walking speeds, two other unexpected peaks appeared in the histograms (Fig.9). The transition $\boldsymbol{D}$ also occurred at one other moment within the stride cycle. In order to fully understand the relationship between symbols and temporal gait measurements, the accelerometer data was compared to mocap data of the same subject walking at normal speed. Stance and swing are clearly identified in Fig.10. By analogy, the swing and stance phases were determined for the accelerometer data as exemplified in Fig.11.
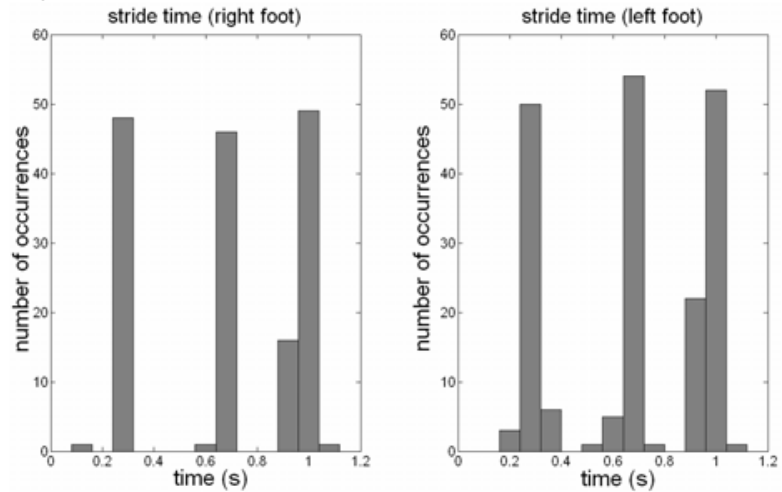

Fig. 9. Occurrence of transition $\boldsymbol{D}$ within the stride cycle. Example taken from normal speed walking data.

The two unexpected peaks in the histogram of transitions $\boldsymbol{D}$ (one around $0.3 \mathrm{~s}$ and the other around $0.7 \mathrm{~s}$ ) do not correspond directly to swing and stance. However, the similarity between the two histograms provides information about the temporal symmetry between the feet. The same is true for all other symbol transitions. A measure of temporal gait symmetry may be derived from the transition histograms as follows (2),

$$
S I_{\text {tran }}=\frac{\sum_{j} \frac{1}{n j}\left|h_{R j}-h_{L j}\right|}{\sum_{j} \frac{1}{n_{j}}\left|h_{R j}+h_{L j}\right|}
$$

where $\boldsymbol{n}_{\boldsymbol{j}}$ is the number of non-empty histogram bins for transition $\boldsymbol{j} ; \boldsymbol{h}_{\boldsymbol{R} \boldsymbol{j}}$ is the normalized transition $\boldsymbol{j}$ histogram for the right foot; and $\boldsymbol{h}_{\boldsymbol{L} j}$ is the normalize transition $\boldsymbol{j}$ histogram for the left foot.

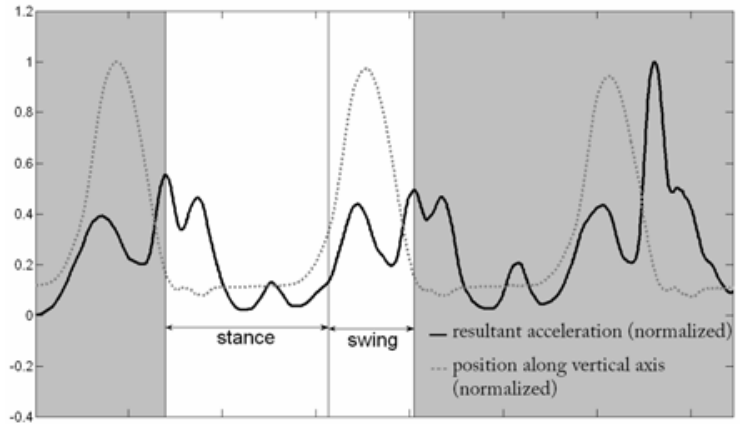

Fig. 10. Mocap data of resultant acceleration $(x, y$, and $z$ axis $)$ and position along the vertical axis. The swing and stance phases are well defined.

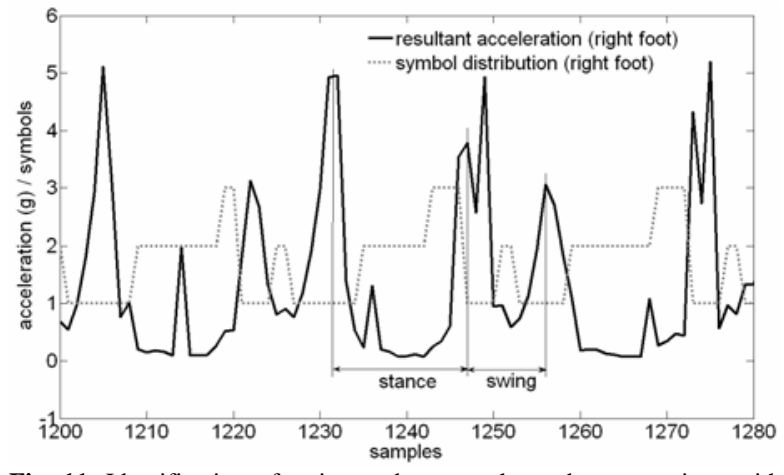

Fig. 11. Identification of swing and stance phases by comparison with mocap data.

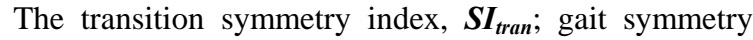
index, $\boldsymbol{S I}$; mean and standard deviation, $\boldsymbol{s t d}$, of stride time for normal, slow and limp walking are presented in Table V. Notice that for $\boldsymbol{S I}$ and $\boldsymbol{S I}_{\text {tran }}$ the lower the absolute value, the greater the symmetry. The negative value of $\boldsymbol{S I}$ indicates that the stride time is greater for the left foot. The

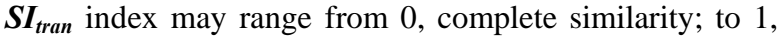
no similarity. Its value is, therefore, easier to interpret than the traditional $\boldsymbol{S I}$ and it may be calculated independent of stride time.

\begin{tabular}{|c|c|c|c|c|c|c|}
\multicolumn{1}{c|}{ Table V. Overview of results } \\
\cline { 2 - 7 } \multicolumn{1}{c|}{} & \multicolumn{2}{c|}{ Normal walk } & \multicolumn{2}{c|}{ Slow walk } & \multicolumn{2}{c|}{ Limp walk } \\
\cline { 2 - 7 } \multicolumn{1}{c|}{} & right & left & right & left & right & left \\
\hline mean (s) & 1.0218 & 1.076 & 1.3422 & 1.3692 & $\mathrm{X}$ & $\mathrm{X}$ \\
\hline std (s) & 0.0366 & 0.0385 & 0.099 & 0.1254 & $\mathrm{X}$ & $\mathrm{X}$ \\
\hline $\boldsymbol{S I}$ & \multicolumn{2}{|c|}{0.4119} & \multicolumn{2}{c|}{-1.9916} & \multicolumn{2}{c|}{$\mathrm{X}$} \\
\hline $\boldsymbol{S I}_{\text {tran }}$ & \multicolumn{2}{|c|}{0.1711} & \multicolumn{2}{c|}{0.3304} & \multicolumn{2}{c|}{0.6860} \\
\hline
\end{tabular}

\section{DISCUSSION}

This work focused on using motion primitives to quantitatively analyze gait unsteadiness, though this methodology may be employed for different purposes.

Consider the following divisions within the field of 
movement analysis: positioning, when an object is tracked through space; posture, when the static posture of and object is determined; and dynamics, when dynamic qualities of the movement are studied e.g. hand gesture classification. The motion-primitive based method presented here may find several applications in the latter group, such as ambulatory monitoring.

In addition, this method may be applied to different sensor signals, from biometric data such as ECG to seismographic data. This method is then able to analyze information about a human subject as well as information about different events happening in a certain environment. The symbolic representation derived in this method is also an effective way of compressing data.

\section{CONCLUSION}

The future demographic changes in the elder population will exert pressure on the health care system to treat agerelated problems. One way to cope with this is to provide intelligent monitoring systems to assist comfortable and safe aging at home. A powerful feature for these systems is the ability to recognize and classify human movements.

Most of the research efforts put into detecting and classifying human movements have so far lacked the ability to generalize motion. Clustering and templatematching techniques are only suitable for detecting few, particular movements. To overcome this problem, the idea of motion primitives was brought forth. This work discussed the viability of deriving a motion language from accelerometer signals to quantitatively analyze gait unsteadiness. A bottom-up linear approach was used for segmentation. The features extracted from each segment were: variability along the $y$ axis; the tangent of the angle between the segment and the horizontal plane; average acceleration along the $y$ axis; and average acceleration along the $z$ axis. These features were used to cluster the segments into 3 symbols. The symbols were used to determine stride time, variability in stride time and temporal symmetry between the two feet.

This first study shows that simple techniques may perform the tasks required for the creation of a motion language capable of describing temporal gait measurements. A more detailed study of segmentation techniques will be carried out in order to improve the performance of the method and provide accurate tools for continuous, unobtrusive, motion analysis.

\section{ACKNOWLEDGMENT}

The authors would like to thank Tommy Salomonsson for engineering support and Lina Lundgren for providing assistance for the mocap measurements.

\section{REFERENCES}

[1] Population Division of the Department of Economic and Social Affairs of the United Nations Secretariat, "World Population Prospects: The 2006 Revision”, http://esa.un.org/unpp.

[2] D. M. Karantonis, M. R. Narayanan, M. Mathie, N. H. Lovell, B. G. Celler, "Implementation of a real-time human movement classifier using triaxial accelerometer for ambulatory monitoring,"
Information Technology in Biomedicine, IEEE Transactions on, vol. 10, no. 1, pp. 156-167, 2006.

[3] R. Camicioli, D. Howieson, B. Oken, G. Sexton, J. Kayes, "Motor slowing precedes cognitive impairment in the oldest old," Neurology, vol. 50, no. 5, pp. 1496-1498, 1998.

[4] R. K. Ibrahim, E. Ambikairajah, B. G. Celler, N. H. Lovell, "Timefrequency based features for classification of walking patterns," Digital Signal Processing, $15^{\text {th }}$ International Conference on, pp. 187-190, 2007.

[5] R. Mayagoitia, J. Lötters, P. Veltink, H. Hermens, ”Standing balance evaluation using triaxial accelerometer," Gait \& Posture, vol. 16, no. 1, pp. 55-59, 2002.

[6] J. Hausdorff, H.K. Edelberg, S. Mitchell, A. Goldberger, J. Wei, "Increased gait unsteadiness in community-dwelling elderly fallers,” Archives of Physical Medicine and Rehabilitation, vol. 78, no. 3, pp. 278-283, 1997.

[7] P. Grabiner, s. Biswas, M. Grabiner, “Age-related changes in spatial and temporal gait variables," Archives of Physical Medicine and Rehabilitation, vol. 82, no. 1, pp. 31-35, 2001.

[8] S. Frenkel-Toledo, N. Giladi, C. Peretz, T. Herman, L. Gruendliger, J. Hausdorff, "Effect of gait speed on gait rhythmicity in Parkinson's disease: variability of stride time and swing time respond differently," Journal of Neuroengineering and Rehabilitation, vol. 2, no. 1, p. 23, 2005.

[9] J. Gage, "Gait analysis: an essential tool in the treatment of cerebral palsy: Applied kinesiology," Clinical orthopedics and related research, no. 288, pp. 126-134, 1993.

[10] M. Whittle, "Gait analysis: An introduction," published by Butterworth-Heinemann Medical, 2002.

[11] N. Troje, "Decomposing biological motion: A framework for analysis and synthesis of human gait patterns," Journal of Vision, vol. 2, no. 5, pp. 371-387, 2002.

[12] R. Mayagoitia, A. Nene, P. Veltink, “Accelerometer and rate gyroscope measurement of kinematics: an inexpensive alternative to optical motion analysis systems,” Journal of Biomechanics, vol. 35, no. 4, pp. 537-542.

[13] M. Mathie, A. Coster, N. Lovell, B. Celler, “Accelerometry: providing an integrated, practical method for long-term, ambulatory monitoring of human movement,” Physiological Measurement, vol. 25, no. 2, pp. R1-R20, 2004.

[14] C. Bouten, K. Westerterp, M. Verduin, R. Kodde, J. Janssen, "Assessment of energy expenditure for physical activity using a triaxial accelerometer," Medicine \& Science in Sports \& Exercise, vol. 26, no. 12, pp. 1516-1523, 1994.

[15] C. Bouten, K. Koekkoek, M. Verduin, R. Kodde, J. Janssen, “A triaxial accelerometer and portable data processing unit for the assessment of daily physical activity,” Biomedical Engineering, IEEE Transactions on, vol. 44, no. 3, pp. 136-147, 1997.

[16] O. C. Jenkins, M. J. Mataric, “Automated derivation of behavior vocabularies for autonomous humanoid motion," Proceedings of the $2^{\text {nd }}$ International Joint Conference on Autonomous Agents and Multiagent systems, pp. 225-232, 2003.

[17] D. Vecchio, R. Murray and P. Perona, "Decomposition of human motion into dynamics based primitives with application to drawing tasks," Automatica, vol. 39, pp. 2086-2098, 2003.

[18] C. Holden, “The origin of speech,” Science, vol. 303, no. 5662, pp. 1316-1619, 2004.

[19] G. Rizzolatti, L. Craighero, “The mirror-neuron system,” Annual Review of Neuroscience, vol. 27, no. 1, pp. 169-192, 2004.

[20] K. Kahol, P. Tripathi, S. Panchanathan, "Documenting motion sequences with a personalized annotation system," Multimedia, IEEE, vol. 13, pp. 37-45, 2006.

[21] P. Fihl, M. Holte, T. Moeslund, L. Reng, “Action recognition using motion primitives and probabilistic edit distance," $4^{\text {th }}$ Conference on Articulated Motion and Deformable Objects, pp. 375-384, 2006.

[22] G. Guerra-Filho, Y. Aloimonos, "A language for human action," Computer, vol. 40, pp. 42-51, 2007.

[23] A. Zinnen, K. V. Laerhoven, B. Schiele, "Towards recognition of short and non-repetitive activities from wearable sensors," European Conference in ambient Intelligence, pp. 142-158, 2007.

[24] E. Keogh, S. Chu, D. Hart, M. Pazzani, "Segmenting time series: a survey and a novel approach," Data Mining in Time Series Databases, 2004.

[25] L. Nolan, A. Wit, K. Dudziñski, A. Lees, M. Lake, M. Wychowañski, "Adjustments in gait symmetry with walking speed in trans-femoral and trans-tibial amputees," Gait and Posture, vol. 17, pp. 142-151, 2003. 